Sommes-nous sortis du $\mathrm{XIX}^{\mathrm{e}}$ siècle ? Le romantisme français comme matrice historiographique

Haben wir das 19. Jahrhundert überwunden?

Have we left the 19th Century?

\title{
Stéphane Zékian
}

\section{(2) OpenEdition}

\section{Journals}

Édition électronique

URL : http://journals.openedition.org/ceg/5473

DOI : $10.4000 /$ ceg. 5473

ISSN : 2605-8359

Éditeur

Presses Universitaires de Provence

Édition imprimée

Date de publication : 1 octobre 2013

Pagination : $33-46$

ISSN : 0751-4239

Référence électronique

Stéphane Zékian, « Sommes-nous sortis du xIx siècle ? Le romantisme français comme matrice historiographique », Cahiers d'Études Germaniques [En ligne], 65 | 2013, mis en ligne le 01 octobre 2019, consulté le 27 novembre 2020. URL : http://journals.openedition.org/ceg/5473 ; DOI : https://doi.org/ $10.4000 /$ ceg.5473 


\title{
Sommes-nous sortis du $\mathrm{XIX}^{\mathrm{e}}$ siècle? Le romantisme français comme matrice historiographique
}

\author{
Stéphane ZÉKIAN \\ CNRS (UMR 5611 / LIRE - Lyon)
}

Dans un article récent, l'historien de la Révolution française Jean-Clément Martin attire l'attention sur l'importance cruciale, et peut-être sous-estimée, des premières années du XIX ${ }^{\mathrm{e}}$ siècle dans l'émergence et la fixation d'un grand récit national. En ce qui concerne la France, estime-t-il, les étapes constitutives de l'historiographie nationale "doivent être réexaminées [...] à partir des écrivains du premier $\mathrm{XIX}^{\mathrm{e}}$ siècle, plus importants que leurs successeurs de la III $^{\mathrm{e}}$ République $^{1} \gg$. Si l'auteur parle ici d'historiographies générales, son propos demeure pertinent dans le cas particulier de l'historiographie littéraire de la France. À la faveur d'études consacrées aux programmes scolaires ou au renouvellement de l'histoire littéraire dans l'Université lansonienne, on a souvent insisté sur le rôle de la III ${ }^{\mathrm{e}}$ République naissante dans la stabilisation du canon littéraire de la France. Utile en ellemême, la focalisation sur le dernier $\mathrm{XIX}^{\mathrm{e}}$ siècle a néanmoins pu conduire à négliger certaines orientations prises au cours des décennies antérieures. Il en est longtemps résulté une relative méconnaissance d'autant plus préjudiciable qu'une part considérable des décisions patrimoniales ayant contribué à forger l'institution littéraire moderne ont été prises longtemps avant l'avènement durable de la République.

De ce point de vue, la « représentation actuelle des classiques du $\mathrm{XIX}^{\mathrm{e}}$ siècle », à laquelle ce colloque nous invite opportunément à réfléchir, délimite un champ problématique de première importance. Elle offre un cadre idéal pour mesurer ce que nous gagnons à embrasser tout le $\mathrm{XIX}^{\mathrm{e}}$ siècle lorsque nous voulons comprendre historiquement, dans une démarche de généalogie critique, selon quelles lignes de force s'est dessinée l'institution littéraire dont nous sommes encore, consciemment ou non, les héritiers.

\footnotetext{
1 Jean-Clément MARTin, « Réflexions sur les évolutions historiographiques depuis le bicentenaire de la Révolution française », French Historical Studies, t. XXXII, n 4, 2009, p. 695.
} 
Avant d'entrer en matière, une rapide mise au point lexicale est nécessaire, l'expression « classiques du XIX ${ }^{\mathrm{e}}$ siècle " revêtant des significations distinctes. En premier lieu, elle désigne les écrivains dont les œuvres, produites au cours du $\mathrm{XIX}^{\mathrm{e}}$ siècle, ont eu les faveurs de la postérité. Typiquement : Victor Hugo, qui fut classicisé de son vivant et bientôt lagarde-et-michardisé. La liste est longue et ne cessera de s'étoffer au fil des manuels d'histoire littéraire publiés tout au long du $\mathrm{XX}^{\mathrm{e}}$ siècle. Mais les classiques du $\mathrm{XIX}^{\mathrm{e}}$ siècle s'entendent également au sens, moins descriptif et plus réflexif, d'auteurs anciens canonisés par le $\mathrm{XIX}^{\mathrm{e}}$ siècle : classiques (aux yeux) du $\mathrm{XIX}^{\mathrm{e}}$ siècle, ceux-là apparaissent dans les grandes synthèses et autres palmarès, depuis La Harpe jusqu'à Lanson. Pour ne prendre qu'un exemple, on a souvent dit que Port-Royal, comme objet culturel propre, était une invention de Sainte-Beuve. Et plus généralement, on pourrait avancer que le " siècle de Louis XIV » est une création du XIX ${ }^{\mathrm{e}}$ siècle (bien que l'expression soit d'ascendance voltairienne).

Distinctes, ces acceptions soulèvent des questions elles-mêmes contrastées. S'interroger sur la représentation actuelle des classiques du $\mathrm{XIX}^{\mathrm{e}}$ siècle au premier sens de l'expression, c'est se demander si nous aimons encore le $\mathrm{XIX}^{\mathrm{e}}$ siècle, si nous dialoguons encore avec lui : lisons-nous encore Victor Hugo ou Lamartine ? Si l'on met en revanche l'accent sur le deuxième sens, on se demandera, non pas si nous aimons encore le $\mathrm{XIX}^{\mathrm{e}}$ siècle, mais, plus radicalement, si nous sommes sortis du XIX ${ }^{\mathrm{e}}$ siècle. Il s'agit alors de savoir si nous pensons encore l'histoire littéraire avec des catégories forgées par les contemporains de Hugo ; ou, pour citer les organisateurs de notre rencontre, de vérifier si « les choix du XIX ${ }^{\mathrm{e}}$ siècle ont en fait jamais été remis en cause ». Le passé du $\mathrm{XIX}^{\mathrm{e}}$ siècle est-il encore le nôtre ? Avons-nous les mêmes ancêtres que les hommes du XIX ${ }^{\mathrm{e}}$ siècle ou y a-t-il eu, depuis lors, rupture d'ascendance ? Répondre à ces questions revient à déterminer dans quelle mesure nous sommes encore des hommes du $\mathrm{XIX}^{\mathrm{e}}$ siècle. Dans l'hypothèse où ces choix auraient été purement et simplement reconduits par le $\mathrm{XX}^{\mathrm{e}}$ siècle, il faudrait alors considérer le $\mathrm{XIX}^{\mathrm{e}}$ siècle, non plus seulement comme un objet patrimonial (à ce titre digne de nos soins philologiques et de notre attention conservatrice), mais aussi et surtout, quoique de manière moins consciente, comme un opérateur patrimonial, comme un prisme traçant avec une autorité jamais questionnée l'horizon de nos héritages culturels.

En réalité, ce que l'on considère comme les « choix du XIX ${ }^{\mathrm{e}}$ siècle» fut d'abord le fait de certains romantiques dont la voix, portant plus loin que d'autres, a su infléchir le cours ultérieur de l'historiographie littéraire nationale. De fait, nous restons largement tributaires d'un grand récit romantique, récit d'autant plus difficile à objectiver que nous en sommes les produits. Or notre manière d'être encore $d u$ XIX $^{\mathrm{e}}$ siècle prend un relief particulier quand on soulève une troisième et dernière acception des " classiques du XIX ${ }^{\mathrm{e}}$ siècle ». Car la formule renvoie aussi, bien sûr, à ceux que l'on a désignés comme tels, souvent à des fins de disqualification, dans le 
cadre de la querelle romantique. Les classiques du $\mathrm{XIX}^{\mathrm{e}}$ siècle, ce ne sont donc pas seulement Racine ou Molière canonisés par la nouvelle culture bourgeoise, ni Hugo fait icône de son vivant : c'est encore toute une constellation de figures moins éclatantes, mais dont le poids historique fut loin d'être insignifiant. Signe de ralliement d'un côté, catégorie de la stigmatisation de l'autre, le classique du XIX ${ }^{\mathrm{e}}$ siècle désigne les gardiens du temple qui, en se faisant les champions de ce que l'on commençait à nommer classicisme, pensaient œuvrer à la conservation des saines doctrines au nom d'un bon sens mis en péril par les revendications tapageuses de l'avant-garde. Le destin posthume de ceux qui répondent à cette troisième acception jette un éclairage cru sur notre dépendance à l'égard de la version romantique du $\mathrm{XIX}^{\mathrm{e}}$ siècle. Quand on considère en effet la (non) postérité de ces gardiens du temple, notre adhésion de fait à la version romantique du $\mathrm{XIX}^{\mathrm{e}}$ siècle prend un relief nouveau. Elle invite à penser que le romantisme s'impose d'abord à nous comme une matrice historiographique.

Or le grand récit romantique, s'il offre une puissante grille de lecture pour appréhender la dynamique du XIX ${ }^{\mathrm{e}}$ siècle, n'en présente pas moins des inconvénients de taille. Sa force de déchiffrement ne doit pas faire oublier le sourd travail de diversion qui fut le sien. Il convient, en conséquence, non de s'en défier pour prendre systématiquement le parti inverse (le but, faut-il le dire, ne sera pas ici de réhabiliter Nisard), mais de mettre en perspective une vulgate encore en circulation, afin d'en explorer les angles morts et d'évaluer la portée historique de leur occultation. Dans cette perspective, la question de la "condition classique " au XIX ${ }^{\mathrm{e}}$ siècle représente un cas d'école. Tout indique, en effet, que notre représentation du classique reste largement inspirée de celle qui fut jadis véhiculée, dans le cadre de la querelle romantique, par les représentants de l'avant-garde. Mais une histoire de la querelle directement calquée sur la version d'un des protagonistes est-elle encore une histoire ? Une représentation élaborée dans le feu de l'action, à des fins d'autolégitimation (et, symétriquement, de déconsidération) ne commande-telle pas une certaine prudence ? Comment ce qui relève d'un argumentaire de combat, d'une stratégie de présentation de soi est-il entré sans réel examen dans l'ordre du discours historique ? En un mot : par quelle ruse de l'histoire l'objet à traiter (le classique du romantique) s'est-il finalement substitué au traitement de l'objet? On se contentera ici de poser quelques jalons, en revenant d'abord sur une case blanche de la mémoire collective, puis en indiquant brièvement ce que nous perdons à ne pas surmonter le complexe ventriloque qui nous fait encore penser et dire l'histoire du XIX siècle avec des mots et une grammaire hérités en droite ligne du romantisme.

\section{Où est passé l'anti-romantisme de gauche ?}

Parmi les fausses évidences que l'historiographie romantique a puissamment accréditées, la catégorisation politique de l'histoire littéraire 
n'est pas la moindre. C'est notamment vrai des querelles, peut-être moins profondes que bruyantes, qui ont scandé le cours du XIX ${ }^{\mathrm{e}}$ siècle. L'interprétation politique de ces querelles s'est souvent soldée par la simplification outrancière de la figure adverse. Quand il théorise la place du romantisme (et la sienne propre) dans le cours de l'histoire, Victor Hugo applique à l'évolution littéraire une critériologie et un répertoire conceptuel inspirés des affrontements politiques de son temps. Ce faisant, il incline à défendre une représentation tendanciellement binaire de la vie littéraire. Tout semble bientôt s'ordonner au grand partage de ce qui avance et de ce qui régresse. Si le romantisme prétend incarner l'esprit d'ouverture et l'idéal d'émancipation, le classique (i.e. le classique selon l'avant-garde) sera forcément assimilé à la crispation d'une posture passéiste. Les exemples sont légion. On connaît ces lignes célèbres de 1830 :

\begin{abstract}
le romantisme, tant de fois mal défini, n'est à tout prendre, et c'est là sa définition réelle, que le libéralisme en littérature. [...] le libéralisme littéraire ne sera pas moins populaire que le libéralisme politique. La liberté dans l'art, la liberté dans la société, voilà le double but auquel doivent tendre d'un même pas tous les esprits conséquents et logiques. ${ }^{2}$ (italiques de l'auteur)
\end{abstract}

Fort du principe, énoncé dans le même passage, que « la liberté littéraire est fille de la liberté politique » (ou encore « une conséquence naturelle, $[\ldots]$ un corollaire immédiat de notre grand mouvement social de $1789 »)$, Hugo affirme que cette certitude s'imposera à l'avenir. Il est vrai qu'il ne ménagera pas ses efforts pour accréditer cette version de l'histoire. Sous le Second Empire, il reviendra à la célèbre "Réponse à un acte d'accusation» de parachever l'autoportrait de l'avant-gardiste en révolutionnaire. Le fameux " bonnet rouge ", qu'il se flattera d'avoir mis au «vieux dictionnaire», résume bien la traduction systématiquement politique des positionnements littéraires à laquelle se livre Hugo.

$\mathrm{Au}$ premier regard, cette solidarité des dimensions politique et littéraire paraît séduisante. Elle présente l'avantage d'être commode, facilement mémorisable. On retient sans peine ce parallélisme des avant-gardes, selon lequel la pointe extrême du mouvement littéraire emboîte le pas et finit même par accélérer l'émancipation politique des sociétés. Le tableau est attrayant. On y résiste si peu que Thibaudet lui donnera plus tard le statut d'une évidence : "On ne peut pas dire précisément que le romantisme soit à gauche. Mais il faut dire qu'il va à gauche. Il va à gauche à partir de 1830, par un brusque changement de direction, comme le Rhône, à partir de Lyon, va au sud $^{3}$. " Image saisissante qui entérine, moyennant la naturalisation de l'histoire littéraire, une vision hugolienne du XIX ${ }^{\mathrm{e}}$ siècle.

\footnotetext{
${ }^{2}$ Victor Hugo, « Sur M. Dovalle» (1830), in Littérature et philosophie mêlées (1834), éd. par Anthony R. W. James, Paris, Klincksieck, 1976, t. 2, p. 131-133. Un large extrait de ce texte sera repris quelques semaines plus tard dans la préface d'Hernani.

3 Albert THIBAUDET, « Romantisme et politique » (1930), in Réflexions sur la politique, éd. par Antoine Compagnon, Paris, Laffont (Bouquins), 2007, p. 407.
} 
Vision engageante s'il en est, mais qui conduit à assécher artificiellement un nombre important de contre-affluents, de canaux et de barrages (pour filer la métaphore de Thibaudet) qui, si l'on en tenait compte, compliqueraient le cours autant qu'ils brouilleraient la limpidité du prétendu fleuve romantique. Les textes réflexifs dans lesquels Hugo théorise sa propre fonction historique sont d'abord des écrits militants. À ce titre, ils sont bien sûr émaillés de silences stratégiques, Hugo détournant le regard (le sien propre, et celui de son lecteur) de tout ce qui fragiliserait la vraisemblance de son scénario. La plus durable de ces distorsions concerne les critiques adressées au romantisme, et à son charismatique chef de file, par les tenants d'une ligne sociale et politique plus farouchement républicaine que la sienne. Pour user d'une formule sans doute trop rapide, c'est bien l'anti-romantisme de gauche qui semble avoir fait les frais d'une historiographie pas toujours émancipée des schèmes hugoliens. La disproportion reste aujourd'hui frappante entre, d'une part, notre bonne connaissance de l'anti-romantisme conservateur (voire réactionnaire) et, d'autre part, la mise en sourdine des critiques adressées au romantisme depuis sa gauche. Nos grilles de lecture enregistrent volontiers l'anti-romantisme des chantres antidémocrates de l'Action française (indéfectibles comme Charles Maurras ou temporaires comme Pierre Lasserre), dont elles font, d'ailleurs bien légitimement, un objet d'analyse à part entière ${ }^{4}$. La contestation venue de l'autre horizon s'avère, en revanche, plus délicate à intégrer. Il n'est d'ailleurs pas indifférent de constater que, même dans le contexte actuel d'une redécouverte de l'antiromantisme, le débordement du romantisme par sa gauche demeure quasiment inaudible ${ }^{5}$.

La matière ne manque pourtant pas. En 1886, La Revue socialiste de Benoît Malon, par ailleurs attachée à la figure de Hugo, publie une réflexion de Jules Bernard sur la position des "lettres devant la plèbe ». Sans être gravement écorné, le prestige du grand homme n'en sort pas tout à fait indemne :

De la pitié, de la pitié, toujours de la pitié ; ils n'ont que de la pitié. La Cosette au sceau des Misérables et le Claude Gueux à la hache : groupes de pitié de ce bonasse bourgeois de génie qui fut Hugo. Tous, tous ; un même vent de charité dédaigneuse presse leurs mots, enfle leur phrase. [...] Hugo parlait au peuple comme aux enfants, avec la même bonté de vieillard à tout petit : une bonté de riche à pauvre. Quand donc leur encre battra-t-elle en mesure avec le sang des

\footnotetext{
4 À titre d'exemple, voir Antoine COMPAGNON, «Maurras critique », Revue d'Histoire Littéraire de la France, 2005/3, p. 517-532 ; Guillaume BOURGEADE, « Romantisme et Révolution chez le jeune Maurras ", in Robert KOPP (dir.), Romantisme et révolution(s). III. Achèvement et dépassement, Paris, Gallimard, 2010, p. 229-262 ; en dernier lieu Michel LEYMARIE, Olivier DARD et Jeanyves GUÉRIN (dir.), Maurrassisme et littérature, Villeneuved'Ascq, Septentrion, 2012.

5 Voir les deux volumes récents coordonnés par Claude MiLLET, Contre le romantisme, Textuel, $\mathrm{n}^{\circ}$ 61, 2010, et Politiques antiromantiques, Paris, Classiques Garnier, 2012.
} 
plèbes ? Quand donc descendront-ils des chaires de Sorbonnes $<\mathrm{sic}>$ ou des marches d'Instituts pour écrire dans la mêlée de la rue, à hauteur du Peuple ?

Cet article n'a rien d'un cas isolé. Son intérêt est au contraire de ramasser en quelques lignes plusieurs décennies de dépit. En 1882, la reprise en fanfare du Roi s'amuse, cinquante ans après la censure exercée par le régime de Louis-Philippe, ne suscite que scepticisme dans L'Égalité de Jules Guesde : la pièce n'est qu'une pantalonnade qui ne doit qu'à la censure l'intérêt qu'elle suscite ; pire, elle fut produite en un temps et dans un milieu « où on avait fait de la littérature un sujet de controverses partiales et de combats ridicules ${ }^{7} \gg$. Le matérialisme soutenu par L'Égalité ne se retrouve évidemment pas dans un ouvrage comme Religions et religion que Hugo avait publié en 1880. À cette occasion, l'organe guesdiste stigmatisait les "rêvasseries creuses et mystiques de son cerveau transcendental $<$ sic $>$ » ainsi qu' « un amas de phrases bien rhythmées $<\mathrm{sic}>$ et harmonieuses peut-être, mais certainement vides $^{8} »$. On dira, et l'on n'aura pas tort, que ces réticences sont peu significatives et qu'on ne saurait les tenir, à cette date tardive, pour une marque d'anti-romantisme. Si elle traduit un renouvellement des grammaires de l'engagement, si elle révèle surtout la profondeur du fossé des générations, l'attaque vise plus particulièrement les derniers feux d'un auteur dont l'œuvre est derrière lui. L'empreinte laissée par Hugo dans les combats de son siècle pour l'émancipation n'est pas ici frontalement mise en cause.

Tel n'avait cependant pas toujours été le cas et il se trouva, tout au long du siècle, des publicistes pour vitupérer la modération d'un avant-gardiste moins militant que beau parleur. L'exemple le moins méconnu reste celui de Paul Lafargue, proche collaborateur de Guesde dans les bureaux de L'Égalité, qui dénonça les faux-semblants d'un bon bourgeois déguisé en homme de progrès. C'est en 1885 , soit au milieu d'un concert d'hommages officiels, que Lafargue, non sans audace, dénonce les raccourcis complaisants et cet art consommé de la publicité mensongère dont résulte à ses yeux, en abusant la crédulité du peuple, "la légende de Victor Hugo ». Selon lui, les proclamations satisfaites du grand homme, façade rhétorique en trompe-l'œil, ne devraient pas faire oublier qu'il tourna toujours son action « en faveur de la "République des affaires" et contre la "République sociale"" ". L'attaque est frontale et n'a cette fois rien d'un dissentiment ponctuel. Ce sont bien la trajectoire générale de Hugo et ses positionnements successifs dans les combats du siècle qui sont ici mis à nu.

${ }^{6}$ La Revue socialiste, t. $4, \mathrm{n}^{\circ} 22$, septembre 1886, p. 811

${ }^{7} L^{\prime}$ Égalité, $4^{\mathrm{e}}$ série, $\mathrm{n}^{\circ}$ 32, 24 novembre 1882, p. 1-2. L'article est de Léon PICARD.

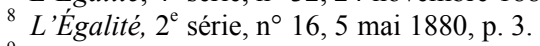

9 Roger FAYOLLE, «Paul Lafargue critique littéraire et propagandiste du matérialisme historique » (1976), in Jacques BerSANI, Michel Collot, Yves JEANNERET et al. (dir.), Comment la littérature vient à nous, Paris, Presses de la Sorbonne nouvelle, 2009, p. 259. Voir Paul Lafargue, Critiques littéraires, éd. par Jean Fréville, Paris, Éditions sociales internationales, 1936. 
Or le reproche est ancien. Entre la révolution de février 1848 et les sanglantes journées de juin, Proudhon n'avait pas eu de mots assez durs pour flétrir ce qu'il tenait pour l'alliance objective des gens de lettres («les notables de la phraséurgie ») et des hommes du pouvoir. À cette occasion déjà, le républicanisme affiché par certains hommes de plume n'était pas, il s'en faut, au-dessus de tout soupçon :

\begin{abstract}
N'est-ce pas le cas de se demander ce qu'il y a de commun entre la révolution et la littérature, ce qu'a fait pour la République, et de quelle utilité peut être à la société, dans l'avenir, cette espèce de parasites vulgairement appelée gens de lettres ? [...] Est-ce la littérature qui a préparé cette révolution ? Est-ce la littérature qui en exprimera le but, les tendances, la loi ? Est-ce la littérature qui viendra la justifier, qui la vengera de ses ennemis ? Quand est-ce que M. Victor Hugo a pris la défense des droits du travail ? Quand est-ce que M. Alexandre Dumas s'est fait connaître par ses idées, par ses mœurs républicaines ? Qu'ontils fait l'un et l'autre, pour la révolution, sinon de calomnier les révolutionnaires ? - Et qu'est-ce qu'ils nous veulent aujourd'hui, ces aligneurs de rimes, ces enfileurs de dialogues? ${ }^{10}$ (italiques de l'auteur)
\end{abstract}

En remontant aux années 1830, dont on ne retient souvent que les attaques d'un Nisard contre « la littérature facile », il faudrait encore faire droit aux réserves des saint-simoniens. Pour n'en citer que quelques-uns, Joncières dans Le Globe enfantinien, Saint-Chéron dans L'Artiste enjoignent Hugo d'arrimer plus solidement sa poésie aux réalités sociales de son temps. Dans Les Étoiles, keepsake saint-simonien publié par Édouard Pouyat, c'est toute l'esthétique du poète qui se trouve battue en brèche ${ }^{11}$. Dans les pages qu'il consacre à cet épisode romantico-publicitaire que fut la bataille d'Hernani, Jean-Marc Hovasse rappelle à juste titre que « les clivages littéraires ne se superposaient pas aux clivages politiques ${ }^{12} »$. Les articles insérés dans $L e$ National par Armand Carrel, républicain de la veille et non du lendemain, le montrent bien : «On assure, ironise alors le journaliste, que la voix puissante du peuple a demandé l'abolition de la césure ; qu'elle s'est élevée contre les unités d'Aristote, comme autrefois contre les gabelles et les droits de mainmorte. Ce peuple est vraiment bien étonnant ${ }^{13}$. » Plus qu'une simple boutade, ce trait en dit long sur le scepticisme d'une partie (aujourd'hui négligée) de la publicité lettrée face à la thèse d'une « heureuse et

\footnotetext{
${ }^{10}$ Pierre-Joseph ProudHON, « Ce que la révolution doit à la littérature » (28 mai 1848), in Idées révolutionnaires, Paris, Garnier frères, 1849, p. 46-51. Voir aussi cette notation de 1843 dans les Carnets, éd. par Pierre Haubtmann, Dijon, Les presses du réel, 2004, p. 26 : «L'école romantique, corrompue, aristocratique, sensuelle $=$ amie du pouvoir $»$.

${ }^{11}$ Paul BÉNICHOU, Romantismes français, Paris, Gallimard, 2004, t. I, p. 747. Pour une immersion dans la nébuleuse saint-simonienne, voir Philippe RÉGNIER, Les Idées et les opinions littéraires des saint-simoniens (1825-1835), Thèse de troisième cycle, Paris III, 1983, 3 vol.

${ }_{12}$ Jean-Marc HovASSE, Victor Hugo. 1. Avant l'exil, 1802-1851, Paris, Fayard, t. I, 2001, p. 432.

${ }^{13}$ Euvres politiques et littéraires d'Armand Carrel, éd. par Émile Littré et Jean-Baptiste Paulin, Paris, Chamerot, 1859, t. V, p. 282.
} 
indissoluble fraternité du libéralisme et du romantisme ${ }^{14} »$. Même Armand Marrast, quoique bien mieux disposé à l'égard de Hugo, refuse tout net $\mathrm{d}^{\prime}$ « assimiler la révolution littéraire à la révolution politique ${ }^{15} »$.

Les critiques de gauche adressées à Hugo ne procèdent pas d'une seule et même logique. L'argumentaire libéral d'un Carrel ne saurait se confondre avec le grief de cécité sociale formulé par certains saint-simoniens. Quand le premier semble réfuter jusqu'à la possibilité même d'un art pour tous hors des règles classiques, les seconds espèrent l'avènement d'un art authentiquement social. Il reste qu'en dépit de leurs dissemblances, ces deux lignes de refus n'ont pas résisté à la lessiveuse de l'historiographie romantique. À cela, plusieurs raisons. Avantageuse pour l'ethos romantique, la solidarité présumée des révolutions politique et littéraire ne fut pas moins confortable pour l'institution des lettres modernes, qui trouva sans peine, dans cette projection héroïque de l'histoire, la matière rassurante d'une autolégitimation. Mais il y a plus. Une autre cause, efficiente dans la deuxième moitié du $\mathrm{XX}^{\mathrm{e}}$ siècle, explique le profond discrédit où tombèrent les revendications jadis élevées au nom de l'art social. Celui-ci, on le voit par exemple chez Paul Bénichou, a en effet pu passer pour l'inquiétante manifestation d'un totalitarisme avant la lettre ${ }^{16}$.

À la faveur d'un faisceau de causes distinctes mais convergentes, c'est ainsi tout un pan de l'échiquier littéraire du $\mathrm{XIX}^{\mathrm{e}}$ siècle qui s'est bientôt vu réduit à une forme durable d'invisibilité. Hommes du passé égarés au $\mathrm{XIX}^{\mathrm{e}}$ siècle ou précurseurs sectaires du dirigisme artistique, les adversaires de Hugo ont vite été rangés sous les rubriques symétriques (mais également compromettantes) d'un conservatisme retardataire ou d'un préjugé social virtuellement criminel. Condescendance, d'un côté, pour ceux qui ratèrent le train de l'histoire littéraire ; peur, de l'autre, devant le spectre d'une mise au pas brutale des écrivains dont la suite de l'histoire ne donnerait que trop d'exemples : tantôt à rebours du sens de l'histoire (comment peut-on ne pas être révolutionnaire au $\mathrm{XIX}^{\mathrm{e}}$ siècle ?), tantôt en avance mais pour le pire (comment peut-on être totalitaire ?), l'anti-romantisme perdait de fait sur tous les tableaux. Son effacement des tablettes (ou sa mémorisation au titre de curiosité folklorique), s'il n'a donc rien de mystérieux, n'en mérite pas moins un regain d'interrogation.

Revenir aujourd'hui sur les effets induits par la souveraineté historiographique d'Hugo ne vise pas, redisons-le, à la réhabilitation

\footnotetext{
${ }^{14}$ Ibid., p. 279. Plus de trente ans après la mort prématurée de Carrel, Hugo ira jusqu'à le rendre personnellement responsable de sa tardive conversion au républicanisme... (HOVASSE, Victor Hugo 1, p. 438). La critique hugolienne n'a pas épargné Carrel. Voir Anne UBERSFELD, Le roman d'Hernani, Paris, Comédie française / Mercure de France, 1985, p. 81-97.

15 Armand MARRAST, "Hernani », L'Athénée : mémorial des sciences, des lettres et des arts, Paris, J. Corréard J ${ }^{\text {ne, }}$, t. I, 1829-[1830], p. 376.

${ }^{16}$ BÉNICHOU, Romantismes, p. 822-825.
} 
mécanique des antiromantiques tombés en oubli ${ }^{17}$. Il paraît fécond, en revanche, d'évaluer les conséquences que la version romantique du romantisme (pour ne pas dire l'histoire des vainqueurs) fit peser sur la problématisation ultérieure de l'histoire littéraire française.

\section{La diversion romantique}

Telle qu'elle est sans relâche et brillamment accréditée par Hugo, la bipolarisation du champ de bataille littéraire relègue dans l'ombre d'autres lignes de fracture dont l'importance n'a pourtant rien de superficiel au début du XIX ${ }^{\mathrm{e}}$ siècle. En ce sens, la querelle romantique apparait bien comme une querelle écran, puisqu'elle cache autant, sinon plus, qu'elle ne donne à voir. L'adoption majoritaire de l'optique romantique a conduit à projeter sur la charnière des $\mathrm{XVIII}^{\mathrm{e}}$ et $\mathrm{XIX}^{\mathrm{e}}$ siècles une grammaire interprétative issue de 1830. Choix délibéré ou réflexe impensé, l'entre-deux siècles a souvent été problématisé au moyen de grilles explicatives forgées en plein XIX ${ }^{\mathfrak{e}}$ siècle. Quand elle n'a pas été purement et simplement négligée, cette période sans nom n'a guère reçu d'autre statut que celui, sans saveur propre, d'étape préparatoire. Terrain d'échauffement des temps à venir, elle valait moins en elle-même que par ce qu'elle paraissait annoncer. Scénariser de façon crédible l'insurrection romantique imposait d'avoir, au préalable, aplani le terrain, en réduisant le tout début $\mathrm{du} \mathrm{XIX}^{\mathrm{e}}$ siècle à une époque platement classique. Les incidences d'un tel finalisme sur l'historiographie du premier $\mathrm{XIX}^{\mathrm{e}}$ siècle ne sont que trop visibles.

Au mitan du siècle, Thiers pourra, en toute vraisemblance, discerner « une double tendance littéraire » dès l'époque impériale : "Les uns, écrira-t-il, voulaient remonter au XVII ${ }^{\mathrm{e}}$ siècle et à l'antiquité, comme à la source de toute beauté ; les autres voulaient demander à l'Angleterre, à l'Allemagne, le secret d'émotions plus fortes ${ }^{18}$. » On comprend vite que la clef 1830 offre une solution d'intelligibilité rétrospective. Véritable passe-partout, elle permet d'atténuer l'étrangeté du moment 1800 en réduisant sans complexe l'inconnu au connu. En 1852, Guizot, lui-même ancien passeur du romantisme en France par sa monographie shakespearienne, dépeindra le Premier Empire comme l'époque d'une «restauration littéraire du XVII ${ }^{\mathrm{e}}$ siècle $^{19}$ ». Ce qui frappe ici, c'est l'écrasement des différences sous la chape d'une seule et même étiquette "classique », comme si la référence au XVII ${ }^{\mathrm{e}}$ siècle suffisait à fédérer un groupe, à le constituer en ensemble cohérent et homogène. Pardelà leurs différences, les descriptions de Thiers et Guizot se signalent par

\footnotetext{
17 Et ce d'autant moins que leurs successeurs ont parfois eux-mêmes fait valoir un droit d'inventaire. Voir, dans le cas de Lafargue, les objections de Claude WILLARD, « Paul Lafargue, critique littéraire », Le Mouvement social, n 59, 1967, p. 102-110, ici p. 108-110.

18 Adolphe THIERS, Histoire du Consulat et de l'Empire, Paris, Paulin, 1849, t. VIII, p. 153.

${ }^{19}$ François GuIZOT, Corneille et son temps. Étude littéraire, Paris, Didier, 1852, p. VI.
} 
une tendance commune à unifier artificiellement les positionnements classiques du premier XIX ${ }^{\mathrm{e}}$ siècle.

On touche ici à une distorsion de longue portée. Sur ce point, les habitudes contractées tout au long du $\mathrm{XIX}^{\mathrm{e}}$ siècle ne se sont pas fondamentalement démenties. Si l'on a tôt pris l'habitude d'étudier, avec force détails, la grande hétérogénéité du mouvement romantique, aucun effort comparable n'a été consenti à l'égard du (prétendu) camp classique. L'objet s'y prêtait pourtant au premier chef, la catégorie classique fusionnant de force des options diverses, parfois inconciliables en matière de politique, d'historiographie et d'épistémologie. C'est dire que la survalorisation du clivage classique/ romantique a contribué à minorer l'enjeu des affrontements, pourtant violents, ayant animé la sociabilité lettrée au tournant des $\mathrm{XVIII}^{\mathrm{e}}$ et $\mathrm{XIX}^{\mathrm{e}}$ siècles. Quand on lit avec attention les journaux et les brochures publiés à partir de Thermidor, on observe bel et bien une bipolarisation de l'espace public. Mais la frontière la plus marquée ne sépare pas alors les novateurs audacieux et les gardiens du temple arc-boutés sur l'Art poétique de Boileau. Elle oppose plutôt, et ces combats sont loin de se recouper, les hommes de lettres fidèles aux Lumières et qui s'efforcent d'en préserver l'héritage aux zélateurs d'un « siècle de Louis XIV » réduit au triomphe du catholicisme. Or ces deux mouvances défendent chacune une version propre de la tradition classique, ces versions s'avérant elles-mêmes indissociables d'une certaine conception de la littérature. Pour les uns, cette tradition est concentrée dans les œuvres les plus alignées du XVII ${ }^{\mathrm{e}}$ siècle, à l'exclusion d'un XVIII ${ }^{\mathrm{e}}$ siècle identifié au triomphe de l'anarchie, de la confusion des valeurs, en un mot, d'un prosaïsme aggravé par la vogue des sciences exactes. Dans le sillage immédiat du Génie du christianisme, les interventions de Joseph Fiévée ou de Jean-Joseph Dussault dans le Mercure ou le Journal des débats vont bien dans ce sens. C'est aussi le cas des textes littéraires de Bonald ${ }^{20}$. Pour les autres, au contraire, l'héritage du XVII ${ }^{\mathrm{e}}$ siècle ne saurait se confondre avec la doctrine catholique. Les productions littéraires contemporaines de Louis XIV leur paraissent essentiellement compatibles avec le legs des Lumières. Pour certains publicistes, les grands auteurs du $\mathrm{XVII}^{\mathrm{e}}$ siècle auraient même amorcé le mouvement d'émancipation et d'aspiration à la liberté qui, dans le cours du $\mathrm{XVIII}^{\mathrm{e}}$ siècle, éclatera au grand jour. Il n'y aurait donc aucune raison, sinon idéologique, de les opposer de manière aussi sèche. Parmi les promoteurs de cette version plus inclusive de la tradition, pensons à Benjamin Constant ou Antoine Jay, futur détracteur des romantiques mais déjà bête noire des classiques ultras $^{21}$.

${ }^{20}$ Stéphane ZÉKIAN, L'Invention des classiques. Le "siècle de Louis XIV 》 existe-t-il ?, Paris, CNRS, 2012, p. 67-93 et p. 235-254.

${ }^{21}$ Benjamin CONSTANT, "Fragment d'un essai sur la littérature dans ses rapports avec la liberté » (c. 1805), in Écrits littéraires : 1800-1813, Tübingen, Max Niemeyer, 1995, p. 495519. Sur Jay et son ironique Conversion d'un romantique de 1830, voir Anthony GLINOER, La Querelle de la camaraderie littéraire. Les romantiques face à leurs contemporains, Genève, Droz, 2008, p. 79-84. 
Pour être grossièrement coupé en deux, le monde lettré ne présente donc pas pour autant la structure que lui prêtera l'historiographie romantique. Quand le publiciste Delalot distingue, en 1805 , ce qu'il appelle les «deux écoles de notre Littérature ${ }^{22}$ ", les innovations contre lesquelles il s'insurge sont celles du XVIII ${ }^{\mathrm{e}}$ siècle. Et il n'y aurait de meilleur antidote, selon lui, que les fortes leçons du «siècle de Louis XIV ». Ce raidissement ne s'estompe pas avec le temps. En 1819, dans les colonnes du Conservateur, journal dirigé par Chateaubriand, le vicomte de Suleau accusera de nouveau le contraste entre les $\mathrm{XVII}^{\mathrm{e}}$ et XVIII ${ }^{\mathrm{e}}$ siècles, voyant même dans la transition de l'un à l'autre une lutte « entre le bien qui finit et le mal qui commence ${ }^{23}$ ».

Ce qui fait débat, on le voit, c'est d'abord le statut du XVIII ${ }^{\mathrm{e}}$ siècle dans l'histoire nationale. Or il ne s'agit pas seulement d'une question de valeur et de hiérarchie littéraires. Ces polémiques engagent plus profondément la définition même de ce que l'on commence à nommer «littérature ». Les innombrables comparaisons entre les deux siècles classiques révèlent, plus qu'un problème de goût, une bataille épistémologique que le démantèlement du système des belles-lettres rend d'autant plus brûlante. Dans ce contexte, les propositions théoriques et pratiques émanant des héritiers du XVIII ${ }^{\mathrm{e}}$ siècle soulèvent l'indignation d'une fraction importante de la publicité lettrée. Que l'on pense à la levée de boucliers suscitée par les possibles prolongements littéraires de la science de l'homme mise en œuvre par le médecin philosophe Cabanis $^{24}$. Appliquée à ces débats, la grille de lecture romantique se révèle en grande partie inopérante. Elle laisse échapper l'essentiel d'un conflit irréductible à la représentation folklorique et tronquée qu'en donneront après coup certains protagonistes. Pire, elle rend insensible à la pluralité des manières de s'instituer continuateur du XVII ${ }^{\mathrm{e}}$ siècle après la Révolution française. Le classicisme de l'Idéologue Cabanis, du polygraphe Cadet de Gassicourt (auteur de satires contre Chateaubriand) ou du publiciste Pierre Chaussard (proche de la Décade philosophique et actualisateur de Boileau) n'a que peu à voir avec celui de Bonald. Envisager leurs écrits sous le seul angle de la querelle romantique contraint à les classer dans le même camp. Si l'on met cependant l'accent sur leurs définitions respectives du fait littéraire, sur la position que chacun assigne à la littérature dans la cartographie des discours, leurs singularités prennent un relief plus conforme à la réalité de ces conflits aujourd'hui oubliés.

Le cas de Marie-Joseph Chénier (pourfendeur d'Atala et meilleur ennemi $\mathrm{du}$ très classique Dussault) illustre bien les effets produits par le placage

\footnotetext{
${ }^{22}$ Le Spectateur français au XIX $X^{e}$ siècle, ou Variétés morales, politiques et littéraires recueillies des meilleurs écrits périodiques (1805-1812), Genève, Slatkine reprints, 1969, t. III, p. 436-441.

${ }_{23}$ Louis de SUlEAU, « Du siècle de Louis XIV et de la Perfectibilité », Le Conservateur, t. IV, 1819, p. 552.

24 Stéphane ZÉKIAN, « Les enjeux littéraires de la science de l'homme. Bonald et Cabanis dans la "guerre des sciences et des lettres" ", in Yves CiTTON / Lise DuMASY (dir.), Le Moment idéologique : littérature et sciences de l'homme, Lyon, ENS, 2013, p. 47-67.
} 
rétrospectif, sur une période dont l'un des enjeux fut la redélimitation de la littérature, d'une définition qui ne s'est imposée que par la suite ${ }^{25}$. En tant que spécialistes des lettres modernes, nous avons tendance à projeter notre conception de la littérature sur une époque où elle ne représentait qu'une des options possibles, qu'une piste ouverte parmi d'autres. Autrement dit, nous plaquons le résultat des débats sur le cours encore incertain de ces mêmes débats. Et le prestige du romantisme 1830 constitue bien l'un des vecteurs les plus efficaces de ce court-circuitage rétrospectif.

À la vue de ces trop rapides éléments, il est difficile de conclure autrement que sur un mode programmatique. Car il reste beaucoup à faire pour fonder un point de vue autonome sur l'histoire littéraire du XIX ${ }^{\mathrm{e}}$ siècle. Tout herméneute de la tradition se trouve pris de fait dans cette dialectique de l'appartenance et de la distanciation à laquelle Paul Ricœur a consacré des pages décisives ${ }^{26}$. Pas moins qu'une autre, la tradition romantique nous met au défi de vivre cette tension sans en figer de force le mouvement dynamique. Or, jusqu'à une date récente, tout indique que le sentiment d'appartenance a, pour une large part, supplanté le geste de distanciation. L'importance conférée à la bataille d'Hernani reste représentative d'une histoire encore à l'unisson de son objet. Certes, la dimension légendaire de l'événement est chose acquise, de même que l'invraisemblance d'une périodisation faisant de 1830 , date bien tardive, son centre de gravité (« on avait tellement besoin d'y croire $^{27}$ », résume Adrien Goetz). Dès les années 1930, Giraudoux ne cachait d'ailleurs pas sa perplexité, allant jusqu'à ne voir dans cette date fétiche qu' ' un événement mondain » mettant aux prises des classiques revendiqués et des classiques qui s'ignorent ( $«$ pour le fond, pas de querelle ${ }^{28} »$ ).

Cette conscience déjà ancienne d'un héritage en grande partie légendaire n'a toutefois pas conduit à en réviser les présupposés ni à en dépoussiérer les angles morts. Les meilleurs indices en sont une méconnaissance persistante des conflits épistémologiques à la charnière du $\mathrm{XIX}^{\mathrm{e}}$ siècle et, corollairement, un relatif désintérêt pour le classicisme progressiste. Il existe un lien sans doute structurel entre, d'une part, notre indifférence aux objections levées par les classiques de gauche contre les prétentions hugoliennes à « révolutionner » la littérature et, d'autre part, l'absence d'une réflexion collective sur les

${ }^{25}$ ZÉKIAN, L'Invention, p. 292-294.

26 Paul RiCCEUR, « Herméneutique et critique des idéologies »(1973), in Du texte à l'action. Essais d'herméneutique. 2, Paris, Le Seuil, 1986, p. 333-377.

${ }^{27}$ Théophile GAUTIER, Histoire du Romantisme, suivi de Quarante portraits romantiques, éd. par Adrien Goetz et Itaï Kovács, Paris, Folio, 2011, p. 23. Voir aussi Agnès SPIQUEL, « La légende de la bataille d'Hernani », in Marie Dollé (dir.), Quel scandale !, Saint-Denis, PUV, 2006, p. 13-27 ; et Myriam ROMAN, «La "bataille" d'Hernani racontée au XIX" siècle : pour une version romantique de la "querelle" ", in Corinne SAMINADAYAR-PERRIN (dir.), Qu'est-ce qu’un événement littéraire ?, Saint-Étienne, PUSE, 2008, p. 125-149.

${ }^{28}$ Jean GIRAUDOUX, «De siècle à siècle. Conférence prononcée à l'occasion du centenaire de Hernani » (1930), in Littérature, Paris, Folio, 1994, p. 182-183. Les critiques de Hugo, rappelle-t-il, étaient « trop heureux de n'avoir pas à protester pour leur bourse et pour leurs habitudes ». 
conditions d'émergence de cette "littérature » dont le XIX ${ }^{\mathrm{e}}$ siècle naissant a tout à la fois redessiné le territoire et réinventé la fonction. Dans Le Sacre de l'écrivain, Paul Bénichou écrit que « [la querelle romantique] a fait oublier celles de l'époque antérieure, qui portaient presque uniquement, entre royalistes et libéraux, sur la préséance à accorder, sans sortir du cadre classique, au $\mathrm{XVII}^{\mathrm{e}}$ ou au XVIII ${ }^{\mathrm{e}}$ siècle, c'est-à-dire au fond sur la condamnation ou l'approbation du ministère progressiste de l'homme de lettres philosophe $\mathrm{e}^{29} »$. C'est peu dire qu'elle les a vouées à l'oubli, et l'auteur lui-même paraît parfois adopter le point de vue romantique. La thèse même du sacre de l'écrivain, miroir gratifiant puisqu'il renvoie l'image d'une littérature au sommet des hiérarchies symboliques et nous érige par là même en héritiers d'une tradition flamboyante, renforce l'illusion d'optique plus qu'elle ne la corrige. Il conviendrait, au contraire, d'insister davantage sur le caractère "compensatoire et défensif ${ }^{30}$ " des discours d'auto-consécration tenus à l'époque. Plus globalement, il est tentant de penser qu'un lien indissoluble noue ensemble le crédit dont jouit encore l'idée d'un sacre de l'écrivain, l'adhésion consciente ou non à la version romantique du romantisme, et l'indifférence commune pour la pluralité des classicismes, en particulier pour les classiques progressistes. Il n'est pas anodin que nombre d'entre eux, à l'image de François Andrieux, Marie-Joseph Chénier ou Victorin Fabre, aient été proches des Idéologues. Rappelons qu'Armand Marrast lui-même, quelques semaines avant d'écrire sur Hernani, faisait encore l'éloge vibrant de cette mouvance philosophique.

Comme le suggère cette proximité avec les Idéologues, eux-mêmes longtemps évincés de la mémoire collective, la mise en sourdine des classiques progressistes est le signe d'une amnésie concernant les querelles de prééminence entre les lettres et les sciences à l'orée du XIX ${ }^{\mathrm{e}}$ siècle. Ce qui disparaît avec eux, c'est un pan essentiel des débats français sur le raccordement des lettres aux autres régimes de discours (ainsi que la question, souvent instrumentalisée à l'époque, d'une virtuelle guerre des lettres et des sciences). Objet d'une curiosité encore marginale, le soubassement doctrinal des débats ponctuant la transition des belles-lettres à la littérature mériterait une approche plus frontale. Ce serait le moyen de déjouer l'anachronisme littéraro-centrique conférant une importance exorbitante au clivage classique/romantique, comme si les divergences poétiques suffisaient à résumer le rapport des forces en présence. Pour ne prendre qu'un exemple, le manque de visibilité dont souffrent encore les enjeux littéraires inhérents aux refontes postrévolutionnaires du monde académique, lieu par excellence d'une réflexion en acte sur l'articulation des discours et la place des écrivains dans le concert des savoirs, trahit une compartimentation dommageable des objets d'analyse. Pour la plupart, ces oubliés de l'histoire luttaient contre la

\footnotetext{
29 BÉNICHOU, Romantismes, p. 260 (je souligne).

30 Alain VAILlAnt, Jean-Pierre BERTRAND et Philippe RÉGNIER, Histoire de la littérature française du XIX $X^{e}$ siècle, $2^{\mathrm{e}}$ éd., Rennes, PUR, 2006, p. 232. Voir aussi Alain VAILLANT, La Crise de la littérature. Romantisme et modernité, Grenoble, ELLUG, 2005, p. 59.
} 
séparation des discours, ils s'efforçaient de pérenniser le modèle encyclopédique légué par les Lumières. Leurs réflexions ne misaient pas tout sur le sacré, elles ne se ressourçaient pas dans une métaphorique religieuse, même laïcisée. Elles posaient néanmoins la question de ce que peut l'écrivain face à la spécialisation des savoirs et, à terme, face à la disciplinarisation des pratiques et des discours.

Or c'est bien à cette injonction disciplinaire que sont aujourd'hui confrontées les lettres. Même si la crise prend maintenant des proportions inquiétantes, ses déterminants ne sont pas radicalement neufs. Les débats sur le savoir de la littérature (et sur la place des études littéraires dans le complexe des discours savants) gagneraient en lisibilité, si l'on se souvenait que la pseudo guerre des cultures a commencé, sous d'autres noms, dès le démantèlement $\mathrm{du}$ système des belles-lettres. D'où l'intérêt de revenir aujourd'hui sur ces perdants de l'histoire littéraire. Quoiqu'inabouti, leur questionnement reste précieux, en ce qu'il oblige à ne pas se reposer sur cette mythologie romantique, rassurante mais illusoire, qui les a condamnés à l'oubli. Leur redonner un peu de visibilité pourrait aider à mieux situer la profonde crise d'identité que traversent les études littéraires. 